\title{
A GROUP OF OPERATIONS ON A PARTIALLY COLORED MAP
}

BY IRVING KITTELL.

It has been previously shown* that if there be a minimum uncolorable map, and it is colored with the exception of one five-sided region in the four colors $A, B, C$, and $D$, then only one of the colors (say $B$ ) will be repeated in the five regions touching the region not colored, and that this ring of regions can be assigned the colors $D B A B C$ in order. It has likewise been shown that when the map is thus colored, there will be two intersecting Kempe chains running from $A$ to $C$ and $A$ to $D$, respectively. We shall say that any map, whether minimum uncolorable or not, which is partially colored in the above manner is colored impasse. The situation may be represented as in Fig. 1.

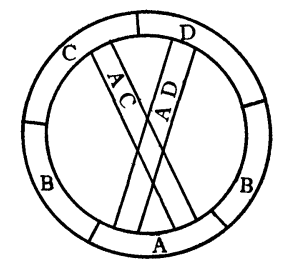

Fig. 1

The diagram does not attempt to show where or how many times the circuits cross one another. It merely means that they do cross. Here we have placed the uncolored five-sided region outside the rest of the map. We do this for compactness, but it must be noted that what follows is not dependent upon this choice of representation, nor upon the choice of lettering. Also to be remembered is the fact that right and left have only arbitrary meanings in discussing a map which may be deformed in any continuous manner over the surface of a sphere. Most writers would draw such a map with the uncolored region inside. We here call the $D$ region the one in the diagram above on the right of $A$, and the $C$ region the one on the left of $A$. We make the following definitions.

The region $A$ is called the vertex of the ring. It is the region in this ring which lies between the two regions having the same color. We call the $A C$ circuit the left-hand circuit and the $A D$ circuit the right-hand circuit. We call the $B D$ chain which includes the $B$ region in the ring between $A$ and $C$ the left-hand

* P. J. Heawood, Map colour theorem, Quarterly Journal of Mathematics, vol. 24 (1890), pp. 332-338. 
chain and the $B C$ chain which includes the other $B$ the righthand chain. We call the $C D$ chain which includes the $C$ and $D$ regions of the ring the end tangent chain; the $B C$ chain touching the ring in the adjacent $B$ and $C$ regions the left-hand tangent chain; and the $B D$ chain touching the ring in the adjacent $B$ and $D$ regions the right-hand tangent chain. Finally, we call the $A B$ chain which includes the vertex, the osculating chain.

We now distinguish nine operations that can be performed on the map above.

$\alpha$ : transposing the colors of the left-hand chain.

$\beta$ : transposing the colors of the right-hand chain.

$\gamma:$ transposing the colors of the left-hand circuit.

$\delta$ : transposing the colors of the right-hand circuit.

$\epsilon$ : transposing the colors of the end tangent chain.

$\zeta$ : transposing the colors of the left-hand tangent chain.

$\eta$ : transposing the colors of the right-hand tangent chain.

$\theta$ : transposing the colors of the osculating chain.

$\iota$ : leaving the coloring unchanged.

In all of these operations no color at all will be assigned to the outside region.

Now, unless each of these operations or any combination of these operations leaves the map in a form analogous to the first form above (that is, with two intersecting circuits running from the region between the two of the same color) it will be possible to color the map. Hence we may say that all of these operations are possible on a minimum uncolorable map. But it does not follow that any map on which all of these operations are possible is necessarily uncolorable.

Errera* describes explicitly only the operation $\alpha$ (or $\beta$ ) of these nine. Here we wish to study as exhaustively as possible all of the operations, and to see if there is any contradiction in their all being possible.

These nine operations are the generators of a group, which we will call the impasse group, and which consists of all possible combinations of these operations. In operating upon a map by two transformations in succession, it is to be noted that the first operation may move the vertex of the ring. Then any second

* Alfred Errera, Du Coloriage des Cartes et de Quelques Questions d'Analysis Situs, Thesis, Gauthier-Villars, Paris, 1921, 66 pp. 
operation is referred to this vertex, for example, $\alpha$ transposes the chain on the left of this new vertex, etc. Thus, the operation $\alpha$ will move the vertex $144^{\circ}$, and hence, if it is performed twice, it will move the vertex through $288^{\circ}$, etc. We may note the following identities:

$$
\iota^{2} \equiv \gamma^{2} \equiv \delta^{2} \equiv \epsilon^{2} \equiv \theta^{2} \equiv \iota, \quad \alpha \beta \equiv \beta \alpha \equiv \iota, \quad \eta \zeta \equiv \zeta \eta \equiv \iota .
$$

It follows that each of the generators has an inverse, and therefore each member of the impasse group has an inverse. Since we are dealing with a map of a finite number of regions, it is apparent that each of the operations of the group is periodic. The operation $\alpha$ has a period of at least twenty, since it can give twenty different arrangements of the colors of the five regions touching the uncolored region. It should be noted that in some maps $\alpha$ may have a period of forty, sixty, etc. The operation $\beta$ also has an apparent period of twenty, while $\zeta$ and $\eta$ have periods of fifteen. The operations $\gamma, \delta, \epsilon, \theta$ all have periods of precisely two.

We do not know how many members of the impasse group of an uncolorable map there may be, but we can show that there must be at least one hundred twenty. There are that many permutations of the colors of the five regions touching the uncolored region, and it can be shown that each one of these permutations can be effected by some operation of the impasse group. We shall not enumerate the operations for the various permutations, but merely assure the reader that such operations have been found. It can be shown that all of the permutations except twelve can be obtained by raising either the primitive operations or operations formed of pairs of the generators to various powers. For these twelve, we can use powers of triples. In all we can use powers of the following operations only: $\alpha, \zeta, \alpha \delta$, $\gamma \alpha, \alpha \gamma, \zeta \alpha, \alpha \zeta, \gamma \eta, \delta \eta, \epsilon \eta, \epsilon \beta \delta, \theta \zeta \gamma, \beta^{2} \epsilon$.

While we thus know that there must be at least 120 members of the impasse group, it must be remembered that there may in some cases be many more. For example, $(\beta \delta)^{8}$ and $(\alpha \gamma)^{7}$ each gives $D A D B C$ for the ring, but the remainder of the map might conceivably be colored differently by these operations.

Errera presented a map on which the operation $\alpha$ could be repeated twenty times to return to the original coloring. At each 
step the coloring remained impasse. We wish to point out a few additional facts about Errera's map, which is shown in Fig. 2. The operations $\alpha$ and $\zeta$ give the following results shown in Figs. 3 and 4.

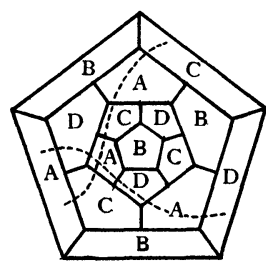

FIG. 2

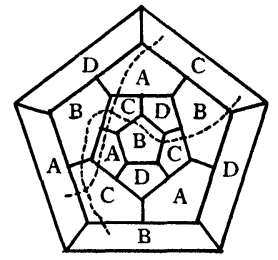

FIG. 3

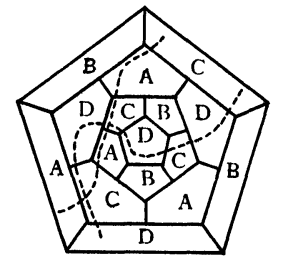

FIG. 4

We say that these operations are in this particular map equivalent, since one coloring can be obtained from the other by simply permuting $A B C D$ to $A D C B$ throughout the whole map. Repetitions of $\zeta$ give the diagrams shown in Figs. 5-7.

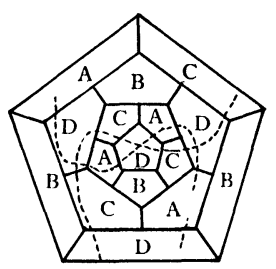

FIG. 5

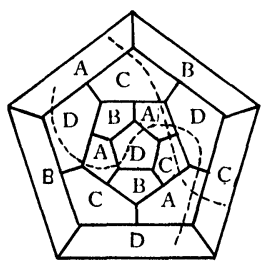

FIG. 6

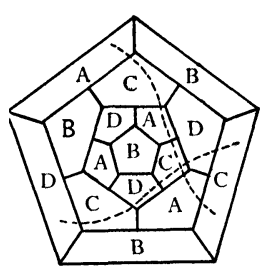

FIG. 7

Now note that $\zeta^{4}$ is equivalent to $\iota$, since this map could have been obtained from the original map by rotating through $144^{\circ}$ and replacing $A B C D$ by $C B D A$. It is apparent, therefore, that $\zeta$ raised to any power is equivalent to $\iota, \zeta, \zeta^{2}$, or $\zeta^{3}$.

Similarly it can be shown that $\alpha^{2}$ is not essentially different from $\zeta^{2}$, nor $\alpha^{3}$ from $\zeta^{3}$ nor $\alpha^{4}$ from $\zeta^{4}$ or $\iota$. Hence any power of $\alpha$ gives an impasse coloring. It can also be seen that $\alpha^{20} \equiv \iota$, and this is what Errera showed.

It can also be shown that $\theta, \gamma, \delta, \beta$, and $\eta$ are equivalent to $\zeta^{2}, \iota, \iota, \zeta^{3}$, and $\zeta^{3}$, respectively; that $\gamma \zeta, \delta \zeta, \beta \zeta, \eta \zeta$, and $\epsilon \zeta$ are equivalent to $\zeta, \zeta, \iota, \iota$, and $\zeta$, respectively; that $\theta \zeta^{2}, \gamma \zeta^{2}, \delta \zeta^{2}, \beta \zeta^{2}$, and $\eta \zeta^{2}$ are equivalent to $\iota, \zeta^{2}, \zeta^{2}, \zeta$, and $\zeta$, respectively; and that $\gamma \zeta^{3}, \delta \zeta^{3}, \beta \zeta^{3}, \eta \zeta^{3}$, and $\epsilon \zeta^{3}$ are equivalent to $\zeta^{3}, \zeta^{3}, \zeta^{2}, \zeta^{2}$, and $\zeta^{3}$, respectively. 
However, the operation $\epsilon$ gives us the diagram shown in Fig. 8. But this coloring is not impasse, since the circuits do not intersect. The operation $\theta \zeta$ gives the diagram shown in Fig. 9;

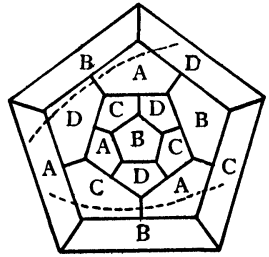

FIG. 8

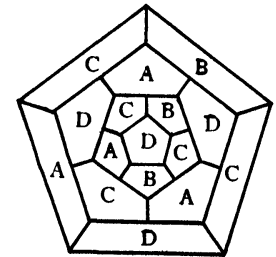

FIG. 9

and this also fails to be impasse since the circuits are both broken. It may be easily shown that $\theta \zeta^{2}$ is likewise ruled out, as is $\epsilon \zeta^{2}$.

In conclusion, we say that the operations $\alpha, \beta, \gamma, \delta, \zeta, \eta$, are possible in any combinations or raised to any power, etc.; that these give rise to only three essentially different colorings (if we note that $\zeta^{3}$ is merely a reflection of $\zeta$ ); and that $\epsilon$ is possible when $\theta$ is impossible and only then, the former being possible on two of the three different colorings but not on the third.

Thus it is shown that there is no possibility of proving any contradiction between $\alpha, \beta, \gamma, \delta, \zeta$, and $\eta$, no matter in what combinations they are used, because in Errera's map we have a case in which they are not contradictory. However, the foregoing suggests that there may be a contradiction between $\epsilon, \theta$, and the other operations. If we can find some operation of the

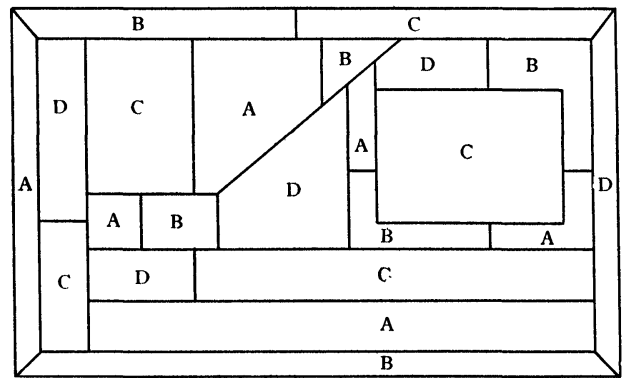

FIG. 10 
impasse group or some set of operations such that no map will remain impasse under these operations, then the four-color problem will be solved.

The map shown in Fig. 10 is such that each of the primitive operations (that is, $\alpha, \beta, \gamma, \delta, \epsilon, \zeta, \eta, \theta)$ leaves the coloring impasse. This shows that no contradiction can be proved for the primitive operations taken singly.

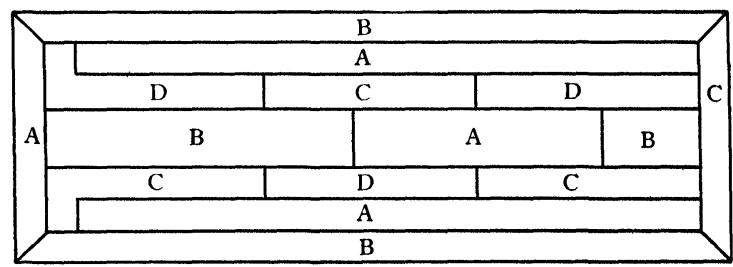

FIG. 11

However, in the map of Fig. 10, $\alpha^{2}$ results in a coloring which is not impasse. Does this mean that there is a contradiction between $\epsilon$ and $\theta$ and $\alpha^{2}$ ? The answer is negative, as is shown by the map of Fig. 11. Here $\epsilon$ and $\theta$ are each possible. Also $\alpha, \alpha^{2}, \alpha^{3}$, and $\alpha^{4}$ are each possible, but $\alpha^{5}$ results in a non-impasse coloring.

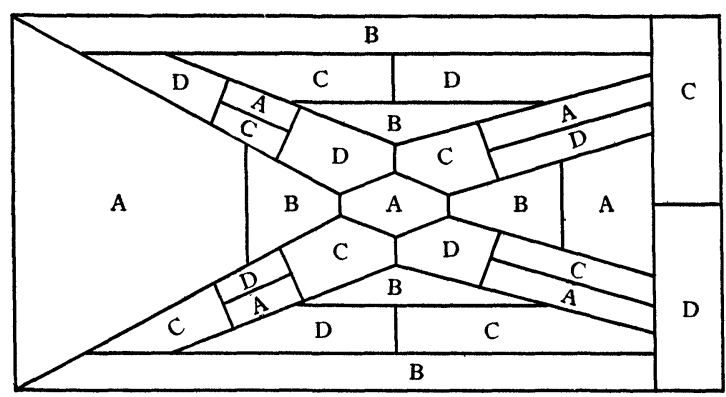

FIG. 12

We now give another map in Fig. 12, on which the operations $\gamma, \delta, \epsilon$ are possible in any combinations. However, in this map none of the other operations (that is, $\alpha, \beta, \zeta, \eta, \theta$ ) are possible. 
But it shows at any rate that any effort to find a contradiction between $\gamma, \delta, \epsilon$, or any combination of these, would be futile.

We have failed to find a map on which all of the operations of the impasse group are possible.

NeW YORK UNIVERSITY

\section{ON THE EQUIVALENCE OF TWO METHODS OF DEFINING STIELTJES INTEGRALS*}

BY B. C. GETCHELL

1. Introduction. The Stieltjes integral, $\int_{a}^{b} \phi(x) d g(x)$, was originally defined for $\phi(x)$ continuous on the closed interval $[a, b]$, and $g(x)$ of bounded variation. The limit which gives rise to this integral is taken as the length of the greatest sub-interval approaches zero. The above restrictions on $\phi(x)$ and $g(x)$, however, are not at all necessary for the existence of the limit, although it fails when the two functions have a common point of discontinuity. A generalization which permits such discontinuities is obtained by taking the limit in the sense of subdivisions, $\dagger$ to be defined below. The Riemann integral is an instance of the first type of limiting process, while the associated Darboux integrals are of the subdivision type. These can be shown to be of the first type as well. It is the purpose of this note to obtain general conditions for the equivalence of the two limits. By the introduction of the notion of interval functions a simple restriction on the integrand is found to be both necessary and sufficient.

2. Subdivisions. By a subdivision, $\sigma$, of the linear interval $X=[a, b]$ will be understood a finite set of adjacent sub-intervals whose sum is $X$. The norm of $\sigma$, the length of the greatest sub-interval, will be written $N_{\sigma}$. By the product, $\sigma^{\prime} \cdot \sigma^{\prime \prime}$, of two subdivisions of $X$ will be understood the subdivision which consists of all products of the form $I^{\prime} \cdot I^{\prime \prime}$, where $I^{\prime}$ is an element of $\sigma^{\prime}$, and $I^{\prime \prime}$ is an element of $\sigma^{\prime \prime}$. It is assumed that every such

* Presented to the Society, April 6, 1934.

$\dagger$ Moore and Smith, $A$ general theory of limits, American Journal of Mathematics, vol. 44 (1922), pp. 102-121. 